\title{
Key Technology of Electronic Nose Gas Recognizer Based on Wireless Sensor Networks
}

\author{
https://doi.org/10.3991/ijoe.v14i10.9304 \\ Mei Hong \\ Changchun University, Changchun, China \\ 13849507 @qq. com
}

\begin{abstract}
Electronic nose gas recognizer is a kind of instrument simulating biological olfactory function for gas detection, which is widely applied in underground construction, aerospace, medical treatment and other fields. The sensing mechanism of the wireless sensor is complex. The wireless sensor array can realize the cross-response of the mixed gas, as well as data acquisition, processing and transmission by wireless transmission. This study applies the wireless sensor array to the electronic nose gas recognition technology, and conducts detection and recognition of three kinds of volatile gas, as well as analyzes the transient response of four wireless sensors and the transient response of wireless sensor array. It is found that the transient response curves are related to the characteristics and sample properties of wireless sensors, but not directly related to sample components. The whole transient response process includes four processes, namely steady state, rising process, maximum response and falling process. The response curve change of wireless sensor array to engine oil volatile gas is similar to that of diesel oil, but the conductance value is smaller than that of diesel oil gas response curve.
\end{abstract}

Keywords-Electronic Nose Gas Recognizer, Wireless Sensor, Transient Response.

\section{Introduction}

Along with the rapid development of artificial intelligence and information technology, the development of artificial intelligence science is inseparable from the acquisition and recognition of various kinds of information [1-2]. Human can acquire information through five physical or chemical sensors of vision, touch, audition, olfaction and taste, among which olfactory sense is of important scientific significance and practical application value [3]. However, some environments are imperceptible to the human's olfaction, so increasingly sensitive analytical instruments are needed [45]. The electronic nose gas recognition technology is a comprehensive recognition technology, which covers wireless sensors, sensors, electronic engineering and recognition modes, and is widely used in many fields such as chemical engineering, medical treatment, environmental monitoring and so on. Gas identification of the electronic nose system is to output test results via gas sensor array, data acquisition pre- 
processing and pattern recognition systems, so the system is more sensitive than human's bio-olfactory system [6-7].

In recent years, with the rapid development of underground construction, manned spaceflight and deep sea exploration engineering, higher and higher requirements have been raised on the safety of non-open space equipment systems [8]. According to incomplete statistics, the explosion caused by flammable and explosive gas leakage is the fourth most serious threat to personal property and system safety [9]. At present, the researches on electronic nose gas recognition system at home and abroad generally includes sensor array technology, sensor array signal pre-processing and feature extraction technology, and signal pattern recognition technology, and the research level is shallow [10-11]. The wireless sensor network is a distributed network which realizes data acquisition, processing and transmission functions through wireless communication and wireless transmission. The application of the wireless sensor network to electronic nose gas recognition will greatly improve its transmission rate and data acquisition capability. The network is featured with fast response, good reversibility and repeatability, and high sensitivity [12]. There are four types of commonly used wireless sensors: resistor type, mass type, optical type and capacitancecharge coupling type [13]. This study applies the wireless sensor array to the electronic nose gas recognition technology, and conducts detection and recognition of three kinds of volatile gas, as well as analyzes the transient response of four wireless sensors and the transient response of wireless sensor array.

\section{Dynamic Modeling of Wireless Sensor Response and Analysis of Transient Response Signal}

\subsection{Dynamic analysis of wireless sensor response}

Based on the sensitivity of electronic nose gas recognition, the transient response information model of wireless sensor is established, and the gas eigenvalue and mixed gas are identified to realize the qualitative and quantitative analysis of gas [14]. The gas sensing mechanism based on wireless gas sensor involves many theories such as material surface morphology, gas sensing material microstructure and gas response dynamics [15-16]. The wireless sensor for gas recognition detects gas by the change in the resistance value of the gas after reaction on the surface of the semiconductor thin film. When the semiconductor thin film contacts a certain molecule, an electron depletion layer is generated on the surface of the material, increasing the resistance [17-18]. A wireless sensor array is essentially different from a single wireless sensor array in operating characteristics. The response of a single wireless sensor to gas is expressed by intensity value while the wireless sensor array can realize a response pattern to types and concentrations of gas. Taking the oxygen ionization model as an example, a reaction will occur as shown in Formula 1:

$\mathrm{O}_{2}{ }^{\text {gas }}+2 \mathrm{e} \leftrightarrow 2 \mathrm{O}^{-}$

The total accumulation rate of surface oxygen ions [O-] is: 
$\frac{d\left[0^{-}\right]}{d t}=\mathrm{k}_{1} \mathrm{P}_{\mathrm{O}_{2}}[\mathrm{e}]_{\mathrm{S}^{-}}^{2}-\mathrm{k}_{-1}\left[\mathrm{O}^{-}\right]^{2}-\mathrm{k}_{\text {react }}^{\mathrm{A}} \mathrm{P}_{\mathrm{A}}\left[\mathrm{O}^{-}\right]$

Where, $\mathrm{k}_{1}$ and $\mathrm{k}_{-1}$ are the rate constants of adsorption and desorption of oxygen ion respectively, which reflect the corresponding excitation energy.

When the wireless sensor contacts with oxygen molecules, the gas pressure $\mathrm{P}_{\mathrm{A}}$ on the film surface suddenly increases. If the initial value is far from the final state, then:

$\mathrm{x}=\mathrm{c}_{3}+\mathrm{c}_{2}+\frac{2 \mathrm{c}_{2}}{\exp \left(2 \mathrm{c}_{1} \mathrm{c}_{2} \mathrm{t}\right)-1}$

Where, $\mathrm{c}_{1}, \mathrm{c}_{2}$ and $\mathrm{c}_{3}$ are all chemical constant parameters.

\subsection{Transient response signal expression and analysis of wireless sensor array}

The relative conductance of the wireless sensor is $y=G / G_{0}$, where $G$ and $\mathrm{G}_{0}$ are respectively the conductance when the wireless sensor is in contact with the gas to be measured and in the initial state, that is:

$y(t)=\frac{\exp \left(2 c_{1} c_{2} t\right)-1}{\left(c_{3}+c_{2}\right)\left[\exp \left(2 c_{1} c_{2} t\right)\right]+c_{2}-c_{3}}$

The steady-state value of relative conductance during the transient response of the wireless sensor is:

$\lim _{t \rightarrow \infty} y(t)=\lim _{t \rightarrow \infty} \frac{\exp \left(2 c_{1} c_{2} t\right)-1}{\left(c_{3}+c_{2}\right)\left[\exp \left(2 c_{1} c_{2} t\right)\right]+c_{2}-c_{3}}=\frac{1}{c_{3}+c_{2}}$

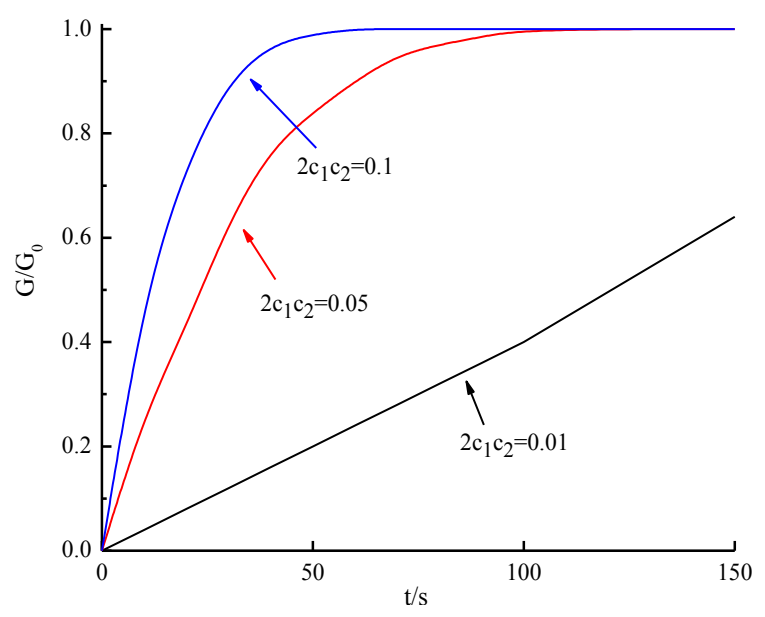

Fig. 1. Wireless sensor transient response simulation value with constant gas concentration

Figure 1 is the simulation value of the transient response of the wireless sensor when the input gas concentration is constant. It is obvious that the response value of the wireless sensor increases rapidly first, then tends to be flat, and finally reaches the 
steady-state value when $2 c_{1} c_{2} \geq 0.05$; the response value of the wireless sensor always increases when $2 c_{1} c_{2}=0.01$.

\section{Design of Electronic Nose System}

\subsection{Hardware design of electronic nose system}

The hardware of the electronic nose system includes keyboard module, temperature and humidity module, wireless sensor array module, wireless sensor signal conditioning module and LCD display module. The hardware system is shown in Figure 2. The measuring circuit of temperature and humidity adopts CHTM-02/A type temperature and humidity sensor module. Figure 3 is the curve of output voltage and relative humidity. It can be seen that the output voltage is positively linear with relative humidity, and the corresponding output voltage is $0-3 \mathrm{~V}$ when the relative humidity is between $0 \%$ and $100 \%$. There are different wireless sensor types for different kinds of sensitive gas. The commonly used sensor types are: TGS813 of $\mathrm{CH}_{4}$ and MQ-7 of CO. Taking the TGS813 sensor of $\mathrm{CH}_{4}$ as an example, the temperature and humidity response curve of the wireless sensor is shown in Figure 4, where Rs represents the resistance value under $1000 \mathrm{ppm} \mathrm{CH}_{4}$ and various temperatures and humidity, and Ro represents the resistance value under $1000 \mathrm{ppm} \mathrm{CH}_{4}$ and $20{ }^{\circ} \mathrm{C} / 65 \% \mathrm{RH}$. The signal conditioning circuit design of wireless sensor should consider the accuracy of output voltage signal and quantization accuracy, and the measurement sensitivity must be maximized when the wireless sensor reaches the transient state.

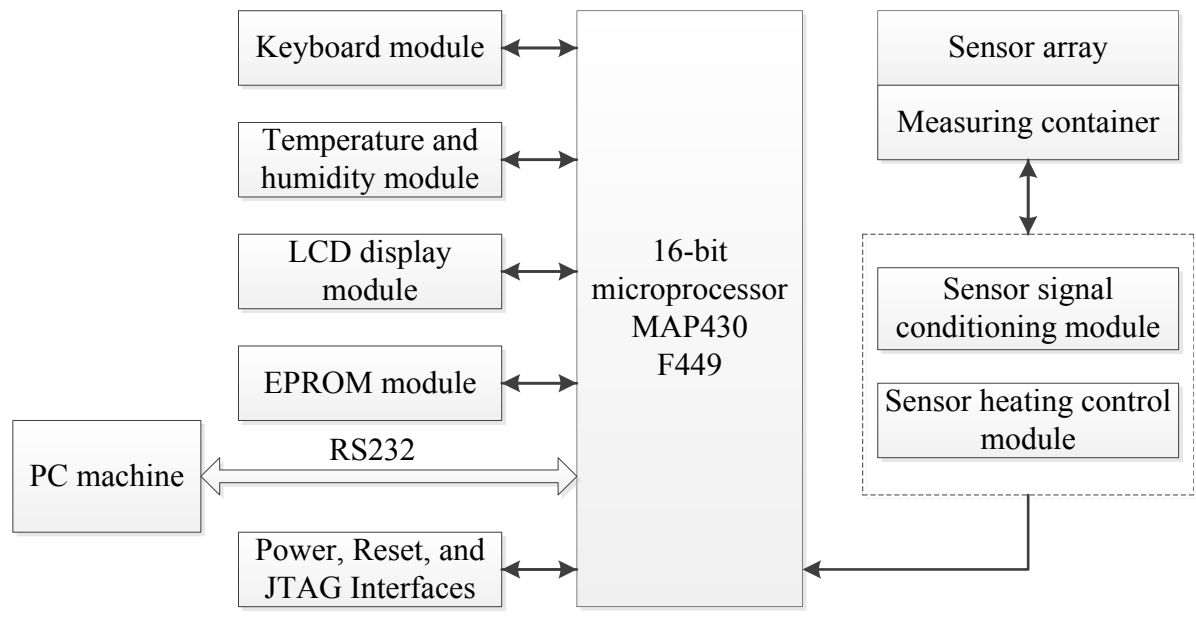

Fig. 2. Electronic nose system hardware block diagram 


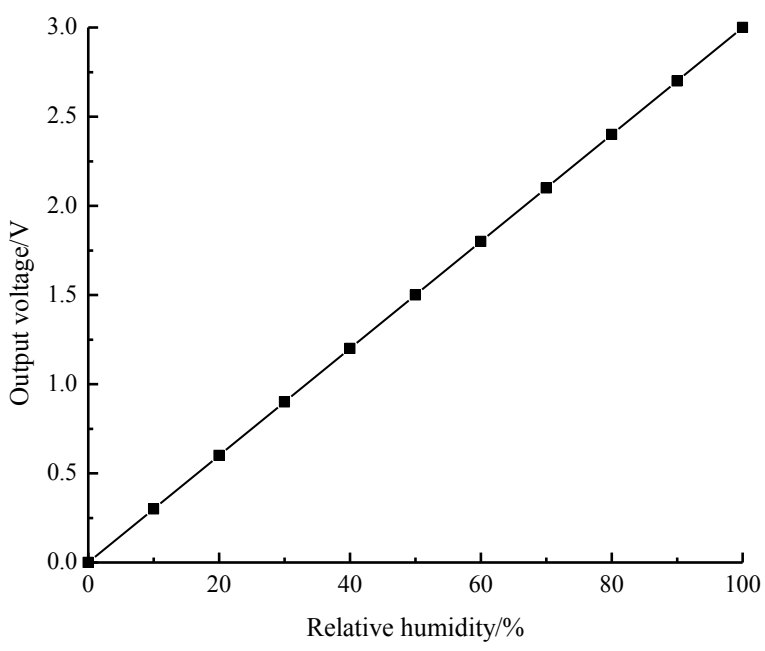

Fig. 3. Output voltage-relative humidity curve

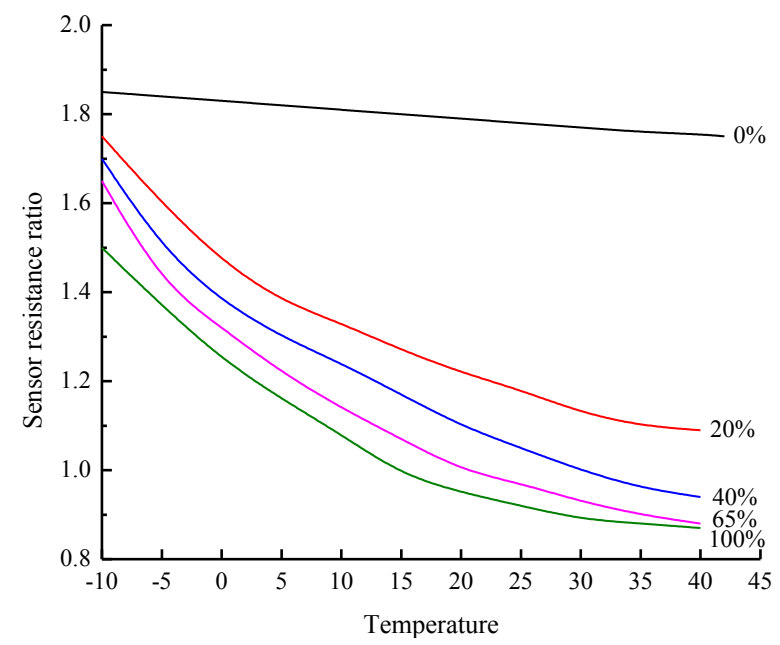

Fig. 4. Temperature and humidity response curve

\subsection{Software design of electronic nose system}

Figure 5 is a block diagram of the electronic nose system software. The whole software design module includes two parts: the design of serial port communication of PC and SCM. The design of PC serial port communication includes measurement interface and data acquisition interface. The SCM serial port communication mainly includes key processing: data transmission, serial port setting, ADC12 conversion and LCD display. The register types related to the ADC12 include conversion control register, interrupt control register, memory register, and memory control register. In the design process of software system, Matlab and $\mathrm{C}++$ programming environment 
are used to realize the science and technology, visualization, storage, openness and expansibility of data. Figure 6 shows Include files path configuration of the edited software system and Figure 7 is the static link library configuration of the system. In addition, the interface design of the software includes the system main interface design, the modal dialog box design, the startup screen design, and design of other subsystems.

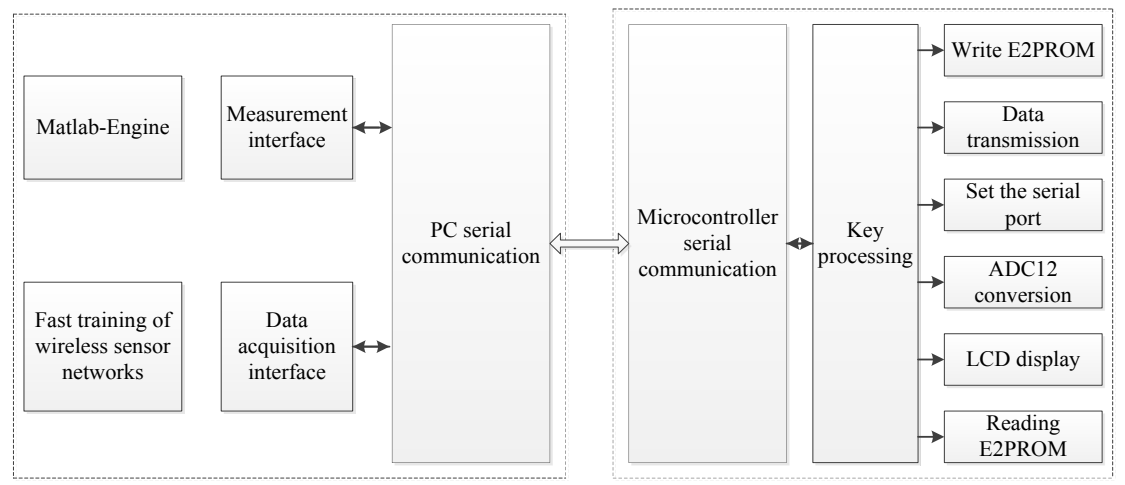

Fig. 5. Electronic nose system software block diagram

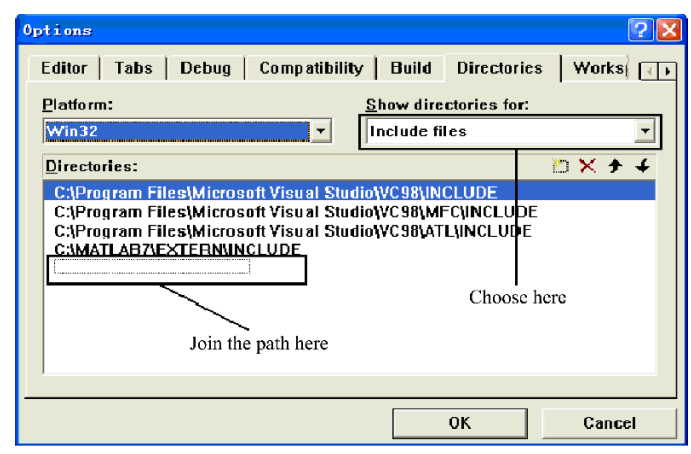

Fig. 6. Include files path configuration

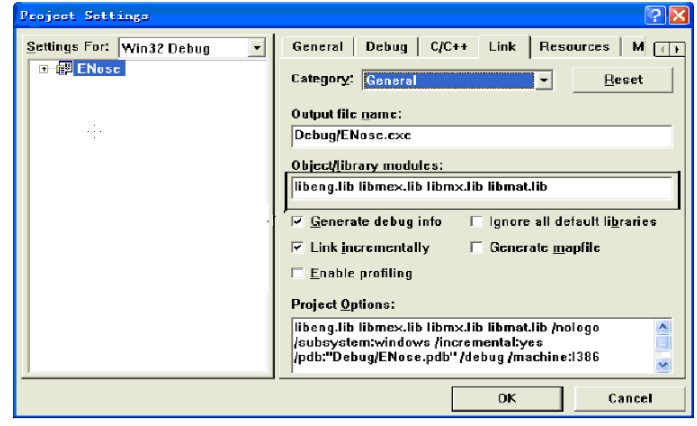

Fig. 7. Static link library configuration 


\section{$4 \quad$ Experiment and Result Analysis}

\subsection{Preparation of experimental samples}

This test selects a portable electronic nose with wireless sensor array, sampling and cleaning channels and data acquisition system, which is highly sensitive and is quick and convenient to test. According to previous studies, the higher the concentration of the gas to be measured, the more the response value $\mathrm{G} / \mathrm{G}_{0}$ deviates from 1 . If the concentration is lower than the limit of detection (LOD) or there is no response gas, the response value $\mathrm{G} / \mathrm{G}_{0}$ is close to or equal to 1 . In the gas environment set in this test, the gas to be measured is charged into the sampling bottle, and the data is collected and transmitted to the computer through the electronic nose gas recognition system with wireless sensor array. The four different sensors have different sensitivity and have very good sensitivity to aromatic compounds, nitrogen oxides, ammonia and hydrogen. The minimum detection volume fraction is $1 \mathrm{ml} / \mathrm{m} 3$. Oil gas from diesel oil, lubricating oil and engine oil is taken as the research object. Diesel oil, lubricating oil and engine oil have different oil components, and the density, color and volatile gas components of oil are different. Therefore, it is far more difficult to recognize and detect the volatility of two kinds of oil gas than that of single component gas. The electronic nose gas recognition response of the wireless sensor array is not only related to the quality of the detection gas, but also related to the temperature, state and environment of the experimental gas. The more components the oil sample contains, the higher the temperature is, and the greater the volatile concentration is. In order to ensure the stability and accuracy of the measurement data of the electronic nose gas recognition system of the wireless sensor array and to eliminate the accumulation effect, the wireless sensor array should be cleaned and standardized before and after each experimental measurement.

\subsection{Gas quantitative analysis based on wireless sensor network}

The transient response of four wireless sensors and the transient response of wireless sensor array are analyzed for the detection and recognition of volatile gas from three kinds of oil. Diesel oil, lubricating oil and engine oil with high and low concentration are selected, marked as D1, Dh, G1, Ch, M1 and Mh. Figure 8 shows the transient response of four wireless sensors to different volatile oil gas. It can be seen that the change rule of each curve is different mainly because the response speed and completeness of the wireless sensors to the measured gas are different, resulting in different response modes and providing different gas recognition information. The relative conductance of wireless sensor changes in the process from initial clean air to sample gas contact. The whole transient response process includes steady state, rising process, maximum response and falling process. When the amount of oil and the concentration of oil are constant, the transient response curve is related to the characteristics and sample properties of the wireless sensor, but not directly related to the sample components. 
To shorten the sensitive time of the wireless sensor, the sampling time is $60 \mathrm{~s}$, and the four distinct response stages are not shown in Figure 8. The four sensors all respond to the three kinds of volatile gas, indicating that the wireless sensor array is cross-sensitive, and the wireless sensor array should be used for testing in complex gas environments instead of a single sensor. Among the four wireless sensors, the response value of the wireless sensor 2 is much larger than that of the other three wireless sensors, and the wireless sensor 1 is sensitive to aromatic compounds.

Figure 9 shows the transient response curve of the wireless sensor array to diesel oil and engine oil volatile gas with low/high concentration, as shown in Figures 9 (a)9 (d). It can be seen that before the wireless sensor 2 and the wireless sensor 4 are in contact with the gas, the relative conductance value $\mathrm{G} / \mathrm{G}_{0}$ increases rapidly, and begins to decrease after reaching the maximum value, and the rate of decrease is less than that of increase. The response curve change of wireless sensor array to engine oil volatile gas is similar to that of diesel oil, but the conductance value is smaller than that of diesel oil gas response curve.
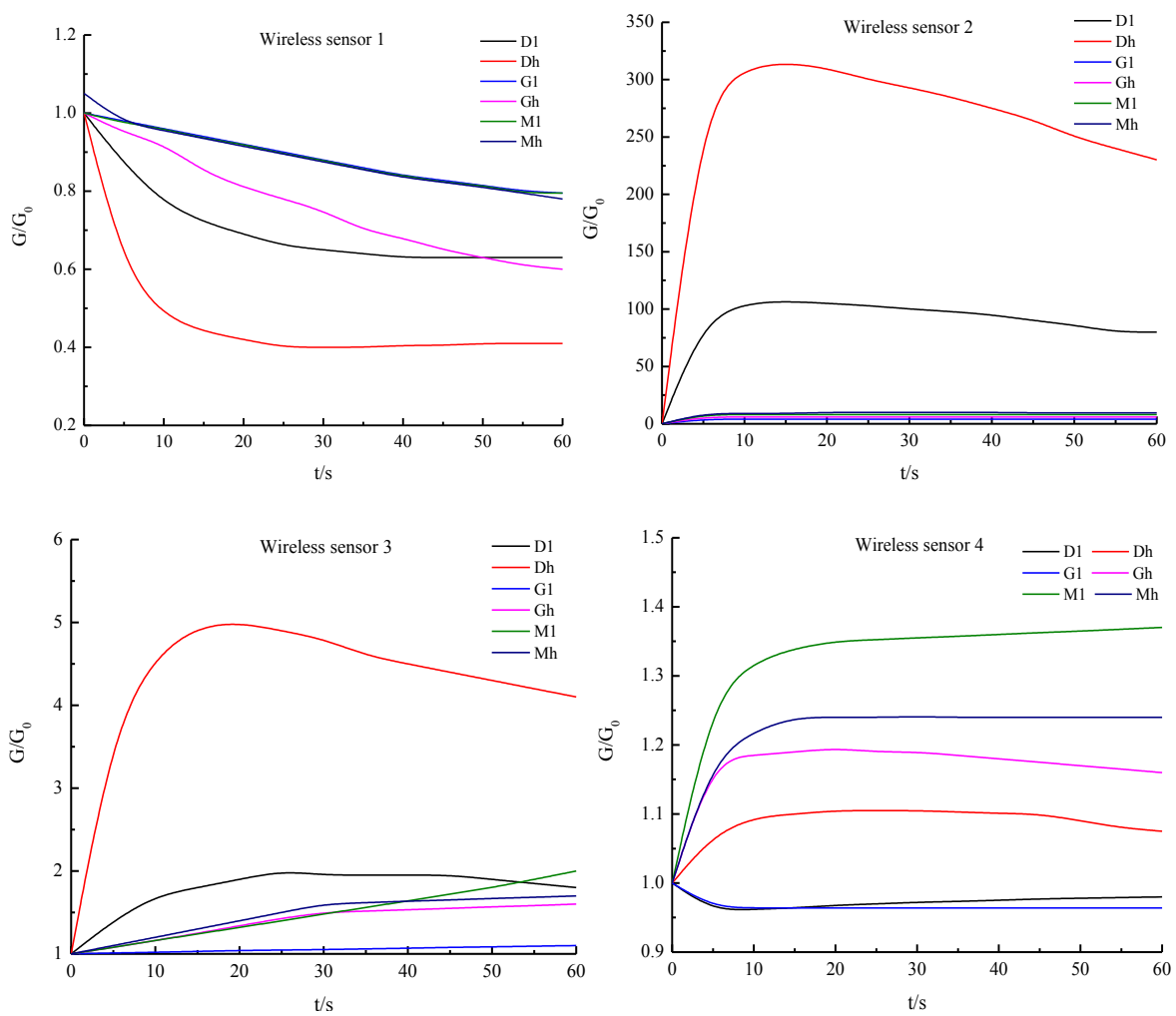

Fig. 8. Figure 8 Transient response curves of four wireless sensors to different volatile oil vapour 


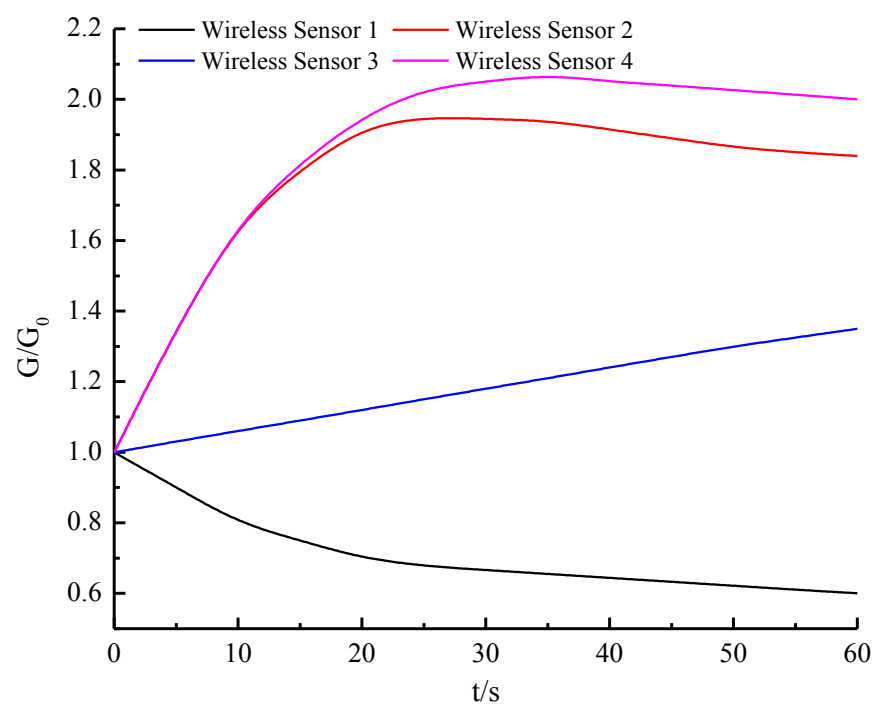

(a)Volatile gas of low concentration diesel oil

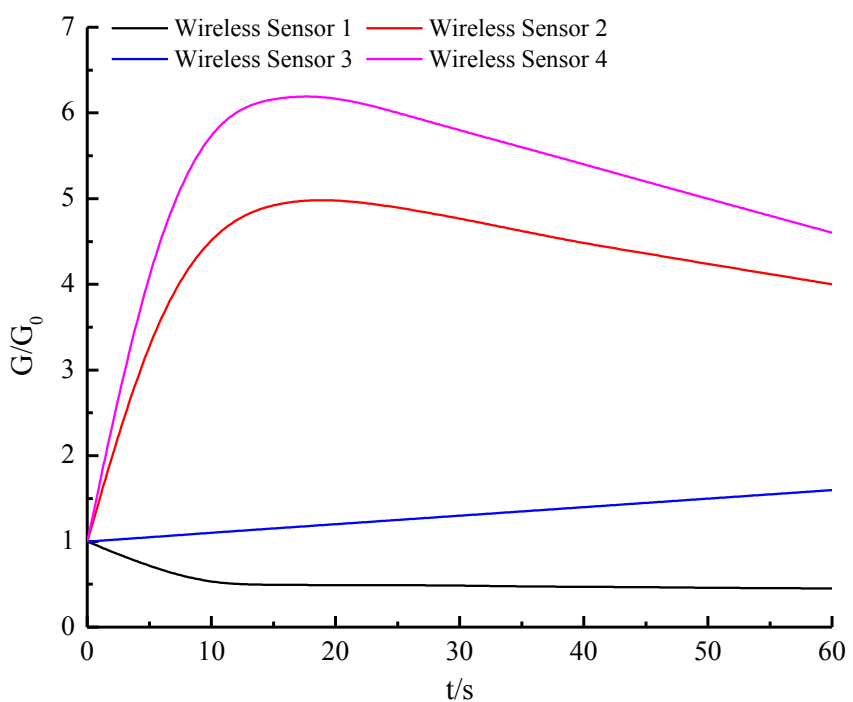

(b)Volatile gas of high concentration diesel oil 


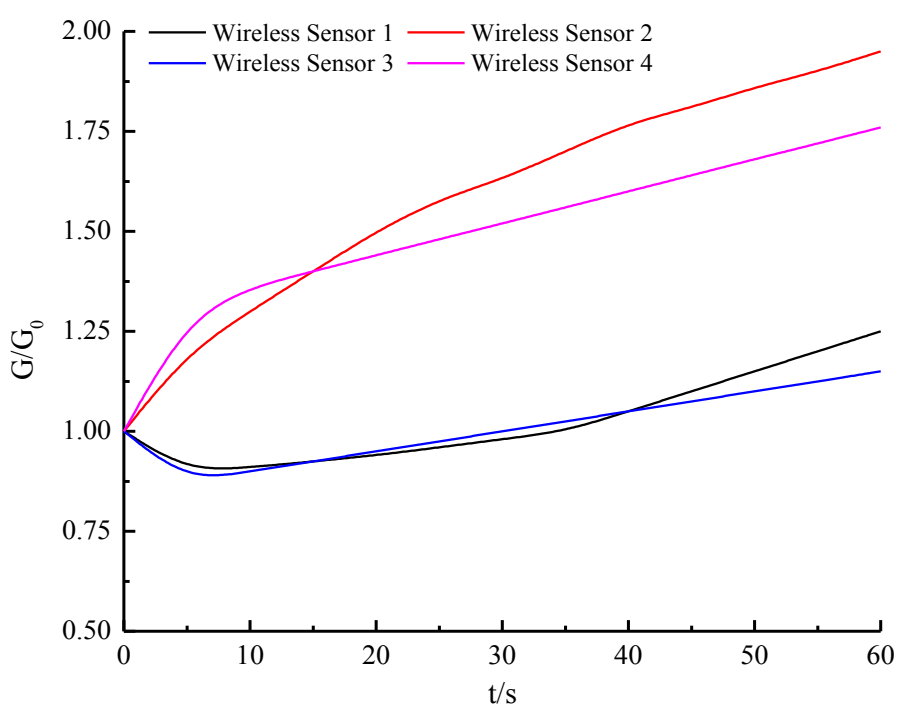

(c)Volatile gas of low concentration oil

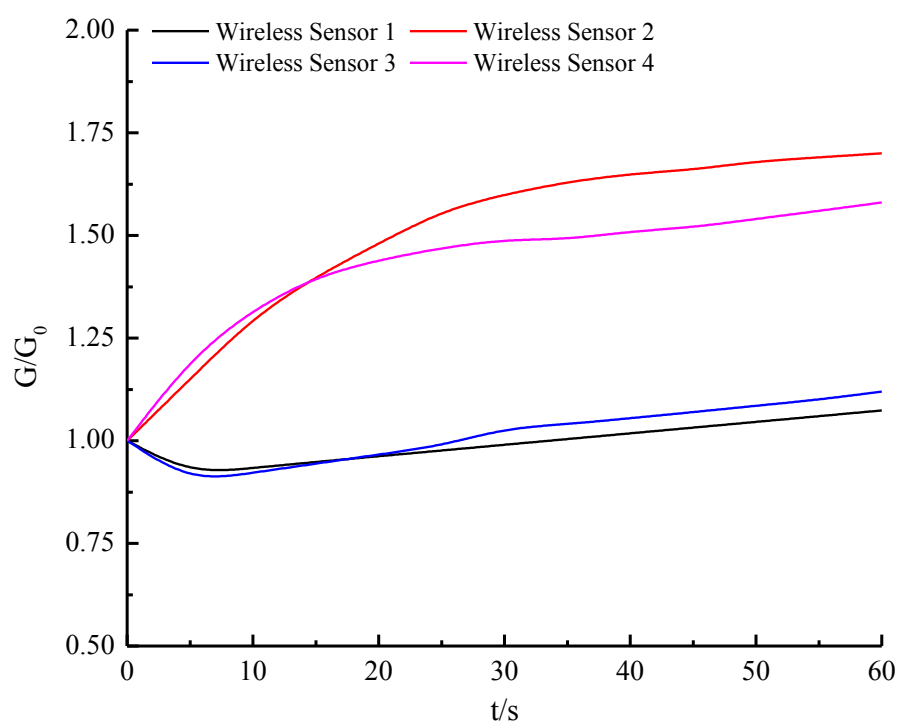

(d)Volatile gas of high concentration oil

Fig. 9. Transient response curve of wireless sensor array to volatile gases in diesel and oil

\section{Conclusions}

This study applies the wireless sensor array to the electronic nose gas recognition technology, and conducts detection and recognition of three kinds of volatile gas, as 
well as analyzes the transient response of four wireless sensors and the transient response of wireless sensor array. The specific conclusions are as follows:

1. The hardware of the electronic nose system includes keyboard module, temperature and humidity module, wireless sensor array module, wireless sensor signal conditioning module and LCD display module. The software design module includes two parts: the design of serial port communication of PC and SCM.

2. The response rate and completeness of the wireless sensor to the measured gas are different, resulting in different response modes. The whole transient response process includes steady state, rising process, maximum response and falling process. The response value of the wireless sensor 2 is much larger than that of the other three wireless sensors.

3. The response curve change of wireless sensor array to engine oil volatile gas is similar to that of diesel oil, but the conductance value is smaller than that of diesel oil gas response curve. Before the wireless sensor is in contact with the gas, the relative conductance value $\mathrm{G} / \mathrm{G}_{0}$ increases rapidly, and begins to decrease after reaching the maximum value, and the rate of decrease is less than that of increase.

\section{Acknowledgment}

This paper is supported by the "13th Five-Year Plan" Scientific Research Planning Project of Jilin Provincial Department of Education "Study on Optimizing Algorithm for Portable Electronic Nasal Sampling Detection". (Contract No.: JJKH20180952KJ).

\section{$7 \quad$ References}

[1] Wang, C., Li, J., Yang, Y., Ye, F. (2018). Combining solar energy harvesting with wireless charging for hybrid wireless sensor networks. IEEE Transactions on Mobile Computing, 17(3): 560-576. https://doi.org/10.1109/TMC.2017.2732979

[2] Singh, A., Awasthi, A.K., Singh, K., Srivastava, P.K. (2016). Modeling and analysis of worm propagation in wireless sensor networks. Wireless Personal Communications, 98(3): 2535-2551. https://doi.org/10.1007/s11277-017-4988-3

[3] Yildiz, H.U., Tavli, B., Kahjogh, B.O., Dogdu, E. (2017). The impact of incapacitation of multiple critical sensor nodes on wireless sensor network lifetime. IEEE Wireless Communications Letters, 6(3): 306-309. https://doi.org/10.1109/LWC.2017.2679744

[4] Cho, J.H., Kim, Y.W., Na, K.J., Jeon, G.J. (2008). Wireless electronic nose system for real-time quantitative analysis of gas mixtures using micro-gas sensor array and neu-rofuzzy network. Sensors \& Actuators B Chemical, 134(1): 104-111. https://doi.org/10.1016/j.snb.2008.04.019

[5] Chowdhury, C., Aslam, N., Ahmed, G., Chattapadhyay, S., Neogy, S., Zhang, L. (2017): Novel algorithms for reliability evaluation of remotely deployed wireless sensor networks. Wireless Personal Communications, 98(11): 1-30.

[6] Herrero, J.L., Lozano, J., Santos, J.P., Suárez, J.I. (2016). On-line classification of pollutants in water using wireless portable electronic noses. Chemosphere, 152: 107-116. https://doi.org/10.1016/j.chemosphere.2016.02.106 
[7] Chansongkram, W., Nimsuk, N. (2016). Development of a wireless electronic nose capable of measuring odors both in open and closed systems is. Procedia Computer Science, 86: 192-195. https://doi.org/10.1016/i.procs.2016.05.060

[8] Aleixandre, M., Santos, J.P., Sayago, I., Cabellos, J.M., Arroyo, T., Horrillo, M.C. (2015). A wireless and portable electronic nose to differentiate musts of different ripeness degree and grape varieties. Sensors, 15(4): 8429-8443. https://doi.org/10.3390/s150408429

[9] Song, K., Wang, Q., Liu, Q., Zhang, H., Cheng, Y. (2011). A wireless electronic nose system using a fe2o3 gas sensing array and least squares support vector regression. Sensors, 11(1): 485-505. https://doi.org/10.3390/s110100485

[10] Brunelli, D., Balsamo, D., Paci, G., Benini, L. (2012). Temperature compensated time synchronisation in wireless sensor networks. Electronics Letters, 48(16): 1026-1028. https://doi.org/10.1049/el.2012.1246

[11] Alaei, M., Barcelo-Ordinas, J. M. (2010). A method for clustering and cooperation in wireless multimedia sensor networks. Sensors, 10(4): 3145-3169. https://doi.org/10.3390/s10 $\underline{0403145}$

[12] Cho, J J., Ding, Y., Chen, Y., Tang, J. (2007). Robust Calibration for Localization in Clustered Wireless Sensor Networks. IEEE International Conference on Automation Science and Engineering, 7(1): 81-95. https://doi.org/10.1109/COASE.2007.4341675

[13] Domenech-Asensi, G., Carrillo-Calleja, J.M., Illade-Quinteiro, J., Martinez-Viviente, F. (2014). Low-frequency cmos bandpass filter for pir sensors in wireless sensor nodes. Sensors Journal IEEE, 14(11): 4085-4094. https://doi.org/10.1109/JSEN.2014.2333538

[14] He, S., Chen, J., Cheng, P., Gu, Y., He, T., Sun, Y. (2012). Maintaining quality of sens-ing with actors in wireless sensor networks. IEEE Transactions on Parallel \& Distrib-uted Systems, 23(9): 1657-1667. https://doi.org/10.1109/TPDS.2012.100

[15] Wei, Z., Xiao, X., Wang, J., Wang, H. (2017). Identification of the rice wines with different marked ages by electronic nose coupled with smartphone and cloud storage platform. Sensors,17(11): 2500. https://doi.org/10.3390/s17112500

[16] Zaoui, F.Z., Hanifi, H.A., Abderahman, L.Y., Mustapha, M.H., Abdelouahed, T., Dja-mel, O. (2017). Free vibration analysis of functionally graded beams using a higher-order shear deformation theory. Mathematical Modelling of Engineering Problems, 4(1): 7-12. https://doi.org/10.18280/mmep.040102

[17] Vito, S.D., Palma, P.D., Ambrosino, C., Massera, E., Burrasca, G., Miglietta, M.L. (2011). Wireless sensor networks for distributed chemical sensing: addressing power consumption limits with on-board intelligence. IEEE Sensors Journal, 11(4): 947-955. https://doi.org/10.1109/JSEN.2010.2077277

[18] Sureshkumar, A., Samson Ravindran, R. (2016). Swarm and fuzzy based cooperative caching framework to optimize energy consumption over multimedia wireless sensor networks. Wireless Personal Communications, 90(2): 1-24. https://doi.org/10.1007/s11277-016$\underline{3274-0}$

\section{Author}

Mei Hong (1981-), female, Research Department of Changchun University, associate researcher. Major research fields: computer science technology, algorithm research, pattern recognition; one article was published in the EI journal, one was in Chinese core journal, and 8 papers were in provincial journals.

Article submitted 21 August 2018. Resubmitted 15 September 2018. Final acceptance 15 October 2018. Final version published as submitted the authors. 